\title{
Mice-lacking LMP2, immuno-proteasome subunit, as an animal model of spontaneous uterine leiomyosarcoma
}

\author{
Takuma Hayashi ${ }^{1,7}$, Akiko Horiuchi ${ }^{2}$, Kenji Sano ${ }^{3}$, Nobuyoshi Hiraoka ${ }^{4}$, Yae Kanai ${ }^{4}$, Tanri Shiozawa ${ }^{2}$, \\ Susumu Tonegawa ${ }^{5}$, Ikuo Konishi ${ }^{6}$ \\ 1 Department of Immunology and Infectious Disease, Shinshu University Graduate School of Medicine, Matsumoto, Nagano \\ 390-8621, Japan \\ 2 Department of Obstetrics and Gynecology, Shinshu University School of Medicine, Matsumoto, Nagano 390-8621, Japan \\ ${ }^{3}$ Department of Laboratory Medicine, Shinshu University Hospital, Matsumoto, Nagano 390-8621, Japan \\ ${ }^{4}$ Pathology Division, National Cancer Center Research Institute, Tokyo 104-0045, Japan \\ ${ }^{5}$ Picower Institution and Department of Biology, Massachusetts Institute of Technology, Cambridge, Massachusetts 02139, USA \\ ${ }^{6}$ Department of Obstetrics and Gynecology, Kyoto University Graduate School of Medicine, Sakyo-ku, Kyoto 606-8501, Japan \\ ${ }^{7}$ Promoting Business using Advanced Technology, Japan Science and Technology Agency (JST), Kawaguchi-shi, Saitama 332- \\ 0012, Japan
}

Correspondence: takumah@shinshu-u.ac.jp

Received June 6, 2010 Accepted July 13, 2010

\begin{abstract}
Uterine tumors are the most common type of gynecologic neoplasm. Uterine leiomyosarcoma (LMS) is rare, accounting for $2 \%$ to $5 \%$ of tumors of the uterine body. Uterine LMS develops more often in the muscle tissue layer of the uterine body than in the uterine cervix. The development of gynecologic tumors is often correlated with female hormone secretion; however, the development of uterine LMS is not substantially correlated with hormonal conditions, and the risk factors are not yet known. Radiographic evaluation combined with PET/CT can be useless in the diagnosis and surveillance of uterine LMS. Importantly, a diagnostic biomarker, which distinguishes malignant LMS and benign tumor leiomyoma (LMA) is yet to be established. Accordingly, it is necessary to analyze risk factors associated with uterine LMS in order to establish a method of treatment. LMP2deficient mice spontaneously develop uterine LMS, with a disease prevalence of $\sim \mathbf{4 0 \%}$ by 14 months of age. It is therefore of interest whether human uterine LMS shows a loss of LMP2 expression. We found LMP2 expression is absent in human LMS, but present in human LMA. Therefore, defective LMP2 expression may be one of the risk factors for LMS. LMP2 is potentially a diagnostic
\end{abstract}

biomarker for uterine LMS, and gene therapy with LMP2encording DNA may be a new therapeutic approach.

KEYWORDS LMP2, uterine leiomyosarcoma, uterine leiomyoma, diagnostic biomarker

\section{INTRODUCTION}

The uterus is a female reproductive organ, located at the center of the pelvis between the left and right ovaries. It is anatomically composed of a lower uterine segment, the uterine cervix serving as a uterine outlet, and an upper uterine segment, the uterine corpus serving as a bag. The uterus, in which the embryo grows, comprises three layers, the uterine endometrium that serves as a bed for the embryo, the myometrium of the wall that protects the embryo, and a serious membrane enveloping the uterus. The myometrium is composed of smooth muscle. In general, the term "uterine tumor" refers to an epithelial malignant tumor of the uterus, which is roughly classified as tumors of the uterine cervix or the uterine body, the former accounting for $~ 70 \%$ of all uterine tumors. Because of the prevalence of screening, cancer of the uterine cervix is decreasing in incidence and usually detected at a very early stage, including stage 0 (intraepithelial cancer). In contrast, cancer of the uterine body is 
increasing in incidence, and rarely detected at the initial stages. While most tumors of the uterine body are adenocarcinomas (derived from the subintimal gland), tumors of the uterine cervix are classified into squamous cancer and adenocarcinoma. The myometrium is composed of smooth muscle. Smooth muscle tumors (SMTs), which develop in the myometrium, have been traditionally divided into benign LMA and malignant LMS based on cytological atypia, mitotic activity and other criteria. Uterine LMS, are of the most common neoplasms of the female genital tract, is relatively rare, having an estimated annual incidence of 0.64 per 100,000 women (Zaloudek and Hendrickson, 2002). Uterine LMS accounts for $2 \%-5 \%$ of tumor of the uterine body and develops more often in the muscle layer of the uterine body than in the uterine cervix (Lin and Slomovitz, 2008; Amant et al., 2009). Distinguishing uterine LMA from uterine LMS is very difficult, and a diagnosis generally requires surgery and cytoscopy.

It has been found that uterine cervix tumors are caused by human papilloma virus in combination with other factors. An infection is established by sexual activity, while a main factor in the development of tumor in the uterine body is the hormonal environment. Patients with uterine body tumors often are unmarried, have never been pregnant, and are taking the use of hormonal agent. High estrogen levels are considered to significantly influence the development of such tumors. Tumor cells developed in the myometrium for some reason gradually become larger due to the influence of the female hormone, estrogen, and generate tumors. However, the precise mechanisms by which uterine LMA and uterine LMS develop are not yet known, and no correlation between the development of uterine LMS and hormonal conditions or other obvious risk factors has been found. The prognosis of uterine LMS is not promising, and the five-year survival rate is approximately $35 \%$, although it depends on disease stage (Lin and Slomovitz, 2008; Amant et al., 2009). It is worth noting that, when adjusted for stage and mitotic count, LMS has a significantly worse prognosis than carcinosarcoma (Miettinen and Fetsch, 2006). As uterine LMS is resistant to chemotherapy and radiotherapy and surgical intervention is virtually the only therapeutic approach (Brooks et al., 2004; Dusenbery et al., 2004; Wu et al., 2006), an efficient adjuvant therapy is required to improve the prognosis of the disease. Although cases accompanied by hypocalcaemia or eosinophilia have been reported, neither clinical abnormality is an initial risk factor for uterine LMS. To identify of the risk factor(s) associated with the development of uterine LMS would significantly contribute to the development of preventive and therapeutic approaches.

\section{BIOLOGICAL ROLES OF THE IMMUNO-PROTEASOME}

When a tissue or an organ is transplanted, the graft is often lost due to an acute rejection caused by the host immunesystem. This is because the cell surface antigens presented by the major histocompatibility complex $(\mathrm{MHC})$ are intrinsic to an individual and differ between the donor and recipient. The immunological self markers on cells surface are the most important immune-system for higher vertebrates, such as mammals, to protect the self from invaders. Proteins are constantly degraded and substituted with newly synthesized forms. Cytoplasmic proteins are mostly degraded by a protease complex, which consists of twenty-eight $20-30 \mathrm{kDa}$ subunits, referred to as the immuno-proteasome (Maniatis, 1999). The proteasomal degradation pathway is essential for many cellular processes, including cell cycle, regulation of gene expression, and responses to oxidative stress. It is also essential for the production of peptide antigens that are presented by MHC class I. Therefore, the immuno-proteasome plays a key role in the presentation of immunological self markers on the cell surface by MHC (Maniatis, 1999). Interferon-y (IFN- $\gamma$ ) is a critical inducing factor for expression and activation of immuno-proteasome in immune-systems (Groettrup et al., 2001). Recent findings have confirmed that IFN-y and lymphocytes prevent primary tumor development, thereby showing a tumor suppressing role in the immune response (Nakajima et al., 2001; Shankaran et al., 2001). IFN-y is crucial in diverse immune events and upregulates large numbers of responsive genes. In addition, expression of the immuno-proteasome's subunits, i.e., low-molecular mass polypeptide (LMP) 2, LMP7, and LMP10, is significantly induced by IFN-y signaling (Gaczynska et al., 1993; Groettrup et al., 2001). The IFN- $\gamma$-inducible proteasomal function is significant in MHC class I-mediated tumor rejection (Delp et al., 2000; Shankaran et al., 2001). Furthermore, molecular biological approaches were used to investigate the correlation of IFN- $\gamma$ with the growth of cancer cells and cancer immunity. A deficiency of IFN-y apparently does not hamper the generation of CTL (Nakajima et al., 2001; Shankaran et al., 2001). Recent reports demonstrated multiple functional deficiencies of components in MHC class I antigen pathway in tumor cells, including LMP2 and TAP-1 signaling (Delp et al., 2000; Shankaran et al., 2001). A possible role for the IFN-Yresponsive gene TAP-1 in tumor recognition was reported (Shankaran et al., 2001). Here, we identified the immunoproteasome subunit LMP2, a single IFN-y-responsive gene product, as obligatory for tumor surveillance (Gaczynska et al., 1993) and demonstrated a tissue-specific role for LMP2 in protection from spontaneous neoplasms of the uterus.

\section{DEVELOPMENT OF MALIGNANT UTERINE TUMOR IN LMP2-DEFICIENT MICE}

Malignant tumors originate from a single cancerous cell and develop as a result of uncontrolled cell proliferation. Malignant tumor cells have properties that are biologically different from those of normal cells. Thus, the host immune-system should 
be able to differentiate malignant tumor cells from the corresponding normal cells. That is, malignant tumor cells present intrinsic antigens (i.e., tumor-cell-specific antigens that can be the targets of immune responses are referred to as "tumor-rejection-antigens (TRAs)") on the cell surface with the assistance of MHC. In many cases, however, almost no reaction by the immune system is observed. Moreover, the incidence of major tumors is not quite different between immuno-deficient (i.e., lymphocyte-deficient) mice and control mice with normal immune systems. Specifically, cancer cells can avoid the immune monitoring system via several ways (Dunn et al., 2002, 2004). Naturally-occurring tumor cells seem to have lost the expression of peptide antigens, TRA, or cell-adhesion factors intrinsic to tumors. Tumor cells may avoid the host immune reaction due to the absence of MHC expression, although no such mechanism has yet been elucidated. However, it is important to demonstrate how tumor cells evade immune-responses in order to prevent the development of tumors.

The genes encoding the subunits of immuno-proteasome, LMP2, LMP7, TAP1 and TAP2, are located in region $\mathrm{H}-2$, which encodes the murine MHC molecule (Fig. 1). LMP2deficient mice show tissue- and substrate-dependent abnormalities in the biological function of immuno-proteasome activity, and impaired activation of the immuno-proteasome in spleen or hepatic cells (Van Kaer et al., 1994). Furthermore, LMP2-deficient mice do not show normal immune responses to virus-infected cells, and such immunopathy is known to result from a failure in the presentation of peptide antigens on the cell surface by MHC (Van Kaer et al., 1994). We found that uterine LMS occurred in female LMP2-deficient mice at 6 months of age or elder, and the incidence at 14 months of age was about $40 \%$ (Fig. 2) (Hayashi and Faustman, 2002). The curve indicating the incidence in mice is very similar to that of human uterine LMS, which occurs after menopause.
Histological examinations of the LMP2-null uterine tumors revealed characteristic abnormalities of LMS (Hayashi and Faustman, 2002). The tumors lacked lymphoid infiltrates, a sign of immune recognition, and consisted of uniform elongated smooth muscle cells arranged into bundles. The nuclei of the tumor cells varied in size and shape, and mitosis was frequent; while the uterine smooth muscle cells of C57BL/6 mice were normal in appearance (Hayashi and Faustman, 2002). Although relatively few Ki-67-positive cells, the proliferating cells of solid tumors, were observed in the basal cell layer of normal uterine smooth muscle, most of the basal cells expressed Ki-67 in LMP2-deficient mice (Hayashi and Faustman, 2002). This immuno-histochemical (IHC) staining indicates abnormal proliferation of the LMP2-deficient cells in the basal layer (Fig. 2) (Hayashi and Faustman, 2002). LMP2-deficient mice that have developed uterine LMS undergo considerable weight loss, and die by 14 months of age (Hayashi and Faustman, 2002). In general, it is not easy to distinguish uterine LMA from uterine LMS. However, in mice, because of such characteristic pathological markers and significant weight loss, a tumor that develops in the uterus of an LMP2-deficient mouse can be considered malignant, i.e., a uterine LMS.

\section{INACTIVATION OF THE IRF-1 TUMOR SUPPRESSOR GENE IN LMP2-DEFICIENT CONDITION}

Uterine LMS was demonstrated to spontaneously develop in 6-month-old LMP2-deficient mice at high frequency. The expression of LMP2 was significantly induced by IFN- $\gamma$ as was the expression of other subunits (Gaczynska et al., 1993; Groettrup et al., 2001). Accordingly, the expression of cellcycle regulators that are regulated by the IFN- $\gamma$ signal cascade or immuno-proteasome activity was examined.

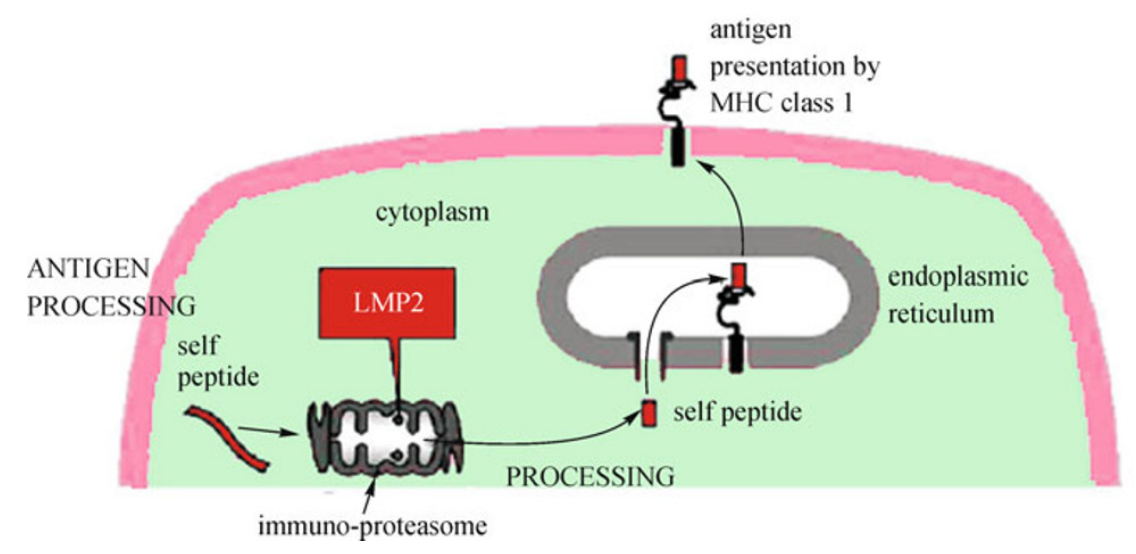

Figure 1. Mediation of proteasomal degradation pathway to antigen presentation by MHC class 1 . The proteasomal degradation pathway is essential for antigen presentation by MHC class 1. Defective LMP2 expression results in tissue- and substrate-dependent abnormality of proteasomal function. Therefore, an impaired proteasome function may promote the initial development of disease, including tumorigenesis. 


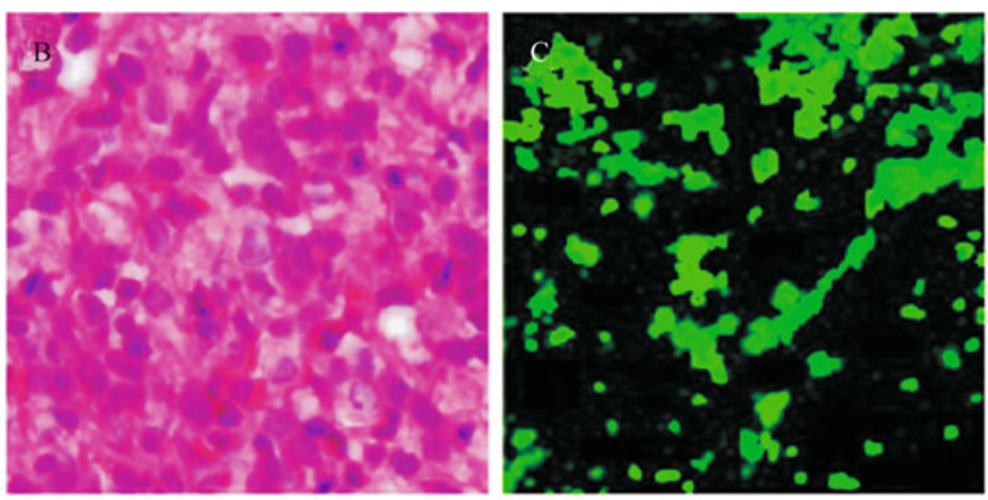

E

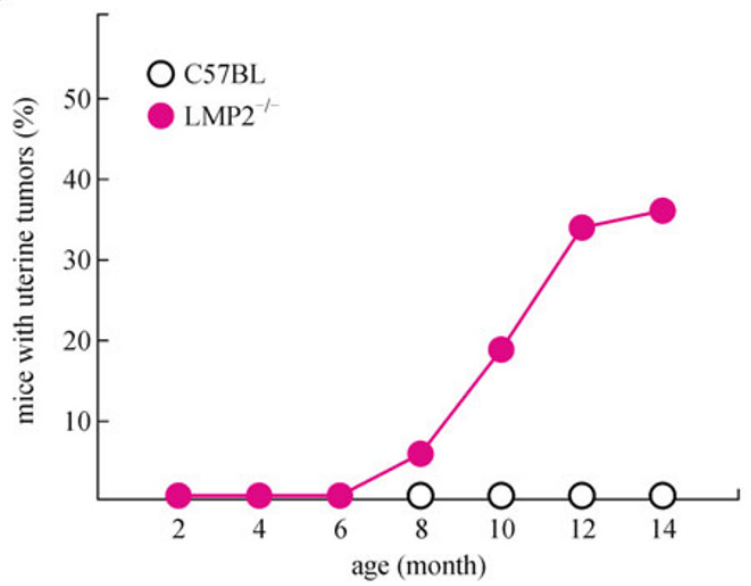

Figure 2. Histological analysis of uterine leiomyosarcoma in LMP2-deficient mice. (A-C) Histological analysis of uterine leiomyosarcoma in LMP2-deficient mice. Among the histological findings of uterine leiomyosarcoma in LMP2-deficient mice, a cytoskeleton, which is characteristic of uterine leiomyosarcoma, is observed ( $B$ and C magnification: 400x). The panel D, in LMP2deficient females, uterine leiomyosarcoma is observed at 6 months of age. The incidence at age 14 months is as high as $40 \%$. (E) The curve indicating the incidence of mouse uterine leiomyosarcoma is very similar that indicating the incidence of human uterine leiomyosarcoma, which is observed after menopause. In mice with tumors of the uterus, significant weight loss is observed. Thus, a tumor that develops in the uterus is diagnosed as malignant, i.e., uterine leiomyosarcoma.

Signal transducer and activator of transcription (STAT) 1 that is activated by IFN-y significantly induced expression of tumor suppressors, such as interferon regulatory factor 1 (IRF1) (Brucet et al., 2004; Hayashi et al., 2006). As a transcriptional regulator, IRF1significantly stimulates LMP2 expression (Brucet et al., 2004; Hayashi et al., 2006). Whether the IFN-Y signal cascade induces the expression of each subunit of the immuno-proteasome, IFR1 and IRF2 in LMP2-deficient mice or the parental strain C57BL/6 was investigated, and no significant difference was observed in the expression of STAT1 or the subunits, LMP7, LMP10, CP9, and IRF2. Moreover, Janus kinase (JAK)-induced phosphorylation of STAT1 stimulated by IFN-y would not be influenced by LMP2 deficiency. However, the expression of the IRF1 tumor suppressor gene was significantly reduced in spleen cells derived from LMP2-null mice in comparison with wild type mice. IRF1 expression in LMP2-null mouse-derived spleen cells was not induced by the IFN-y signal cascade. In addition, wild type-mouse embryonic fibroblasts (MEFs) that had been treated with the proteasome inhibitor MG-132 exhibited reduced IFN- - -inducibility, reproducing a phenotype of the LMP2-deficient mouse. Accordingly, the transcription of IRF1 mRNA depends on activation of the immunoproteasome and is considered to involve the formation of a STAT1 homodimer. Recent reports suggest that proteasomal function contributes to mRNA transcriptional activation (Yanagi et al., 2000; Irina Lassot et al., 2007).

Primary culture of tumor cells (LMP2-UC) was established from the uterine LMS of LMP2-deficient mice, and IRF1overexpressing tumor cells (LMP2-UC-IRF1) were further established by genetic engineering. The LMP2-UC-IRF1 cells were intracutaneously transplanted into immuno-deficient mice (BALB/c nu/nu), and significant inhibiting effects of IRF1 on the proliferation of tumor cells were observed 
(Harada et al., 1993; Hayashi et al., 2006). Thus, a reduced level of IRF1 resulting from a LMP2-deficiency seemed to be a risk factor for uterine LMS in LMP2-null mice. The effects of IRF1 on the proliferation of tumor cells are achieved through the expression of $\mathrm{p} 21^{W A F}$ cell-cycle inhibitors (inhibiting transition from the $\mathrm{G} 1$ to $S$ stage) (Tanaka et al., 1996). Whether or not $\mathrm{p} 21^{\text {WAF }}$ expression or activation is affected in LMP2-deficient mice should be further examined. The tumor suppressor retinoblastoma $(\mathrm{Rb})$ is phosphorylated by a complex of cyclin E/cyclin dependent kinase 2 (CDK2) and then inactivated (Sherr, 2000). In addition, the activity of CDK2 is negatively regulated via degradation of cyclin $E$ by the 26S proteasome (Koepp et al., 2001; Matsumoto et al., 2004). A low level of phosphorylated-Rb is observed in MEFs of LMP2-deficient mice, and the activity of CDK2 for phosphorylation is stronger than that in normal MEFs. In the case of uterine LMS in LMP2-deficient mice, inactivation of the IRF1 or Rb tumor suppressor gene is considered to be significantly involved in cellular transformation and cell proliferation (Fig. 3).

\section{PERSPECTIVES}

Uterine LMS mainly develops in the uterine smooth muscle or endometrial stroma and menstrual anomalies, such as hypermenorrhea and prolonged menstruation; symptoms, such as abnormal hemorrhage, hypogastric pain, lumbar pain and abdominal strains, are observed (Miettinen and Fetsch, 2006). Since these symptoms are same as in uterine LMA, which is assumed to affect $25 \%$ adult females, it is very difficult to differentiate LMA from LMS. Part of uterine LMA becomes malignant and develops into uterine LMS. In gynecological cancers, such as breast cancer, female hormonal imbalance is often a risk factor. As in the case of uterine LMA, however, a correlation between the development of uterine LMS, the female hormone and hormone receptors has yet to be elucidated (Zhai et al., 1999; Akhan et al., 2005). Uterine LMS often develops in the individuals who have been exposed to radiation in the pelvis; other risk factors have not been identified due to the absence of a suitable animal model. The LMP2-deficient mouse is the first model in which uterine LMS develops spontaneously (Hayashi and Faustman, 2002). Defective LMP2 expression may be one of the causes to develop uterine LMS (Hayashi et al., 2006). To demonstrate whether LMP2 is a potential biomarker to distinguish LMS from LMA, we are investigating the reliability and characteristic of LMP2 as diagnostic biomarker with the several clinical research facilities. The clinical researches are not concluded yet, the large scale clinical studies are required. In some cases, uterine LMA may become malignant and develop into uterine LMS. Accordingly, the correlation between the inactivation of LMP2 and the development of uterine LMA remains to be examined. Studies using geneexpression profiling of several known pro-oncogenic factors as well as other factors, such as brain-specific polypeptide PEP-19 and a transmembrane tyrosine kinase receptor, c-kit, are associated with the pathogenesis of uterine LMS (Kanamori et al., 2003; Wang et al., 2003; Ylisaukko-oja et al., 2006). However, merely comparing the expression of potential pro-oncogenic factors between normal and malignant tissues is not sufficient because the results obtained may be the consequence of malignant transformation, and therefore, may not be necessarily the cause. The causal relationship between the inactivation of LMP2 or IRF1 and the development of uterine LMS is currently being examined in vitro and in vivo. Since no spontaneous development of

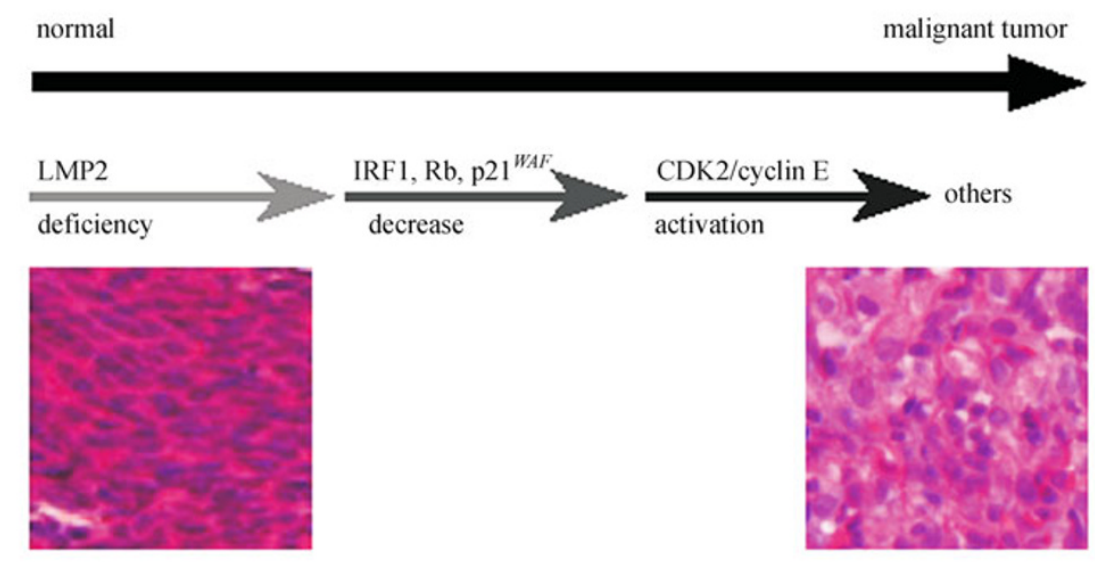

Figure 3. Model of the mechanism for development of uterine leiomyosarcoma. In LMP2-deficient cells, levels of the antioncogenic factor IRF-1, p21 WAF are significantly reduced, and phosphorylated Rb (inactive form) is observed. Cell cycle regulatory factors, CDK2/cyclin E are markedly activated. The inactivation of such tumor suppressors is considered to transform LMP2-deficient cells into malignant tumor cells. 
uterine LMS is observed in IRF1- or Rb-deficient mice, the lack of LMP2 is largely associated with the expression of other known or unknown cell-cycle regulatory factors. Clarification of the correlation between these factors and the development of uterine LMS and the identification of specific risk factors may lead to the development of new methods for diagnosis at genetic level. Uterine LMS is refractory to chemotherapy and has a poor prognosis. The molecular biologic and cytological information described from LMP2-deficient mice contribute remarkably to the development of prevention, diagnosis and therapeutic approaches.

\section{ACKNOWLEDGEMENTS}

We sincerely thank Professor Luc Van Kaer (Vanderbilt University Medical Center). This study was supported in part by grants from the Ministry of Education, Culture, Science and Technology, and The Foundation of Osaka Cancer Research, The Ichiro Kanehara Foundation of the Promotion of Medical Science and Medical Care, Foundation for Promotion of Cancer Research, Kanzawa Medical Research Foundation, The Shinshu Medical Foundation, and Takeda Science Foundation.

\section{ABBREVIATIONS}

IRF1, interferon regulatory factor 1; JAK, Janus kinase; LMA, leiomyoma; LMS, leiomyosarcoma; MEFs, mouse embryonic fibroblasts; MHC, major histocompatibility complex; Rb, retinoblastoma; SMTs, smooth muscle tumors; TRAs, tumor-rejection-antigens

\section{REFERENCES}

Akhan, S.E., Yavuz, E., Tecer, A., lyibozkurt, C.A., Topuz, S., Tuzlali, S., Bengisu, E., and Berkman, S. (2005). The expression of Ki-67, p53, estrogen and progesterone receptors affecting survival in uterine leiomyosarcomas. A clinicopathologic study. Gynecol Oncol 99, 36-42.

Amant, F., Coosemans, A., Debiec-Rychter, M., Timmerman, D., and Vergote, I. (2009). Clinical management of uterine sarcomas. Lancet Oncol 10, 1188-1198.

Brooks, S.E., Zhan, M., Cote, T., and Baquet, C.R. (2004). Surveillance, epidemiology, and end results analysis of 2677 cases of uterine sarcoma 1989-1999. Gynecol Oncol 93, 204-208.

Brucet, M., Marqués, L., Sebastián, C., Lloberas, J., and Celada, A. (2004). Regulation of murine Tap1 and Lmp2 genes in macrophages by interferon gamma is mediated by STAT1 and IRF-1. Genes Immun 5, 26-35.

Delp, K., Momburg, F., Hilmes, C., Huber, C., and Seliger, B. (2000). Functional deficiencies of components of the MHC class I antigen pathway in human tumors of epithelial origin. Bone Marrow Transplant 25, S88-S95.

Dunn, G.P., Bruce, A.T., Ikeda, H., Old, L.J., and Schreiber, R.D. (2002). Cancer immunoediting: from immunosurveillance to tumor escape. Nat Immunol 3, 991-998.

Dunn, G.P., Old, L.J., and Schreiber, R.D. (2004). The immunobiology of cancer immunosurveillance and immunoediting. Immunity 21, 137-148.

Dusenbery, K.E., Potish, R.A., and Judson, P. (2004). Limitations of adjuvant radiotherapy for uterine sarcomas spread beyond the uterus. Gynecol Oncol 94, 191-196.

Gaczynska, M., Rock, K.L., and Goldberg, A.L. (1993). Y-interferon and expression of $\mathrm{MHC}$ genes regulate peptide hydrolysis by proteasomes. Nature 365, 264-267.

Groettrup, M., Khan, S., Schwarz, K., and Schmidtke, G. (2001). Interferon- $\gamma$ inducible exchanges of $20 \mathrm{~S}$ proteasome active site subunits: why? Biochimie 83, 367-372.

Harada, H., Kitagawa, M., Tanaka, N., Yamamoto, H., Harada, K., Ishihara, M., and Taniguchi, T. (1993). Anti-oncogenic and oncogenic potentials of interferon regulatory factors-1 and -2 . Science 259, 971-974.

Hayashi, T., and Faustman, D.L. (2002). Development of spontaneous uterine tumors in low molecular mass polypeptide-2 knockout mice. Cancer Res 62, 24-27.

Hayashi, T., Kobayashi, Y., Kohsaka, S., and Sano, K. (2006). The mutation in the ATP-binding region of JAK1, identified in human uterine leiomyosarcomas, results in defective interferon-gamma inducibility of TAP1 and LMP2. Oncogene 25, 4016-4026.

Kanamori, T., Takakura, K., Mandai, M., Kariya, M., Fukuhara, K., Kusakari, T., Momma, C., Shime, H., Yagi, H., Konishi, M., et al. (2003). PEP-19 overexpression in human uterine leiomyoma. Mol Hum Reprod 9, 709-717.

Koepp, D.M., Schaefer, L.K., Ye, X., Keyomarsi, K., Chu, C., Harper, J.W., and Elledge, S.J. (2001). Phosphorylation-dependent ubiquitination of cyclin $\mathrm{E}$ by the SCFFbw7 ubiquitin ligase. Science 294, 173-177.

Lassot, I., Latreille, D., Rousset, E., Sourisseau, M., Linares, L.K., Chable-Bessia, C., Coux, O., Benkirane, M., and Kiernan, R.E. (2007). The proteasome regulates HIV-1 transcription by both proteolytic and nonproteolytic mechanisms. Mol Cell 25, 369-383.

Lin, J.F., and Slomovitz, B.M. (2008). Uterine sarcoma 2008. Curr Oncol Rep 10, 512-518.

Maniatis, T. (1999). A ubiquitin ligase complex essential for the NFkappaB, Wnt/Wingless, and Hedgehog signaling pathways. Genes Dev 13, 505-510.

Matsumoto, Y., and Maller, J.L. (2004). A centrosomal localization signal in cyclin $\mathrm{E}$ required for $\mathrm{Cdk} 2$-independent $\mathrm{S}$ phase entry. Science 306, 885-888.

Miettinen, M., and Fetsch, J.F. (2006). Evaluation of biological potential of smooth muscle tumours. Histopathology 48, 97-105.

Nakajima, C., Uekusa, Y., Iwasaki, M., Yamaguchi, N., Mukai, T., Gao, P., Tomura, M., Ono, S., Tsujimura, T., Fujiwara, H., et al. (2001). A role of interferon- $\gamma$ (IFN- $\gamma$ ) in tumor immunity: $T$ cells with the capacity to reject tumor cells are generated but fail to migrate to tumor sites in IFN- $\gamma$-deficient mice. Cancer Res 61, 3399-3405.

Shankaran, V., Ikeda, H., Bruce, A.T., White, J.M., Swanson, P.E., Old, L.J., and Schreiber, R.D. (2001). IFNgamma and lymphocytes prevent primary tumour development and shape tumour immunogenicity. Nature 410, 1107-1111.

Sherr, C.J. (2000). The Pezcoller lecture: cancer cell cycles revisited. Cancer Res 60, 3689-3695.

Tanaka, N., Ishihara, M., Lamphier, M.S., Nozawa, H., Matsuyama, T., Mak, T.W., Aizawa, S., Tokino, T., Oren, M., and Taniguchi, T. (1996). Cooperation of the tumour suppressors IRF-1 and p53 in response to DNA damage. Nature 382, 816-818.

Van Kaer, L., Ashton-Rickardt, P.G., Eichelberger, M., Gaczynska, M., Nagashima, K., Rock, K.L., Goldberg, A.L., Doherty, P.C., and 
Tonegawa, S. (1994). Altered peptidase and viral-specific T cell response in LMP2 mutant mice. Immunity 1, 533-541.

Wang, L., Felix, J.C., Lee, J.L., Tan, P.Y., Tourgeman, D.E., O’Meara, A.T., and Amezcua, C.A. (2003). The proto-oncogene c-kit is expressed in leiomyosarcomas of the uterus. Gynecol Oncol 90, 402-406.

Wu, T.I., Chang, T.C., Hsueh, S., Hsu, K.H., Chou, H.H., Huang, H.J., and Lai, C.H. (2006). Prognostic factors and impact of adjuvant chemotherapy for uterine leiomyosarcoma. Gynecol Oncol 100, 166-172.

Yanagi, S., Shimbara, N., and Tamura, T.A. (2000). Tissue and cell distribution of a mammalian proteasomal ATPase, MSS1, and its complex formation with the basal transcription factors. Biochem Biophys Res Commun 279, 568-573.

Ylisaukko-oja, S.K., Kiuru, M., Lehtonen, H.J., Lehtonen, R., Pukkala, E., Arola, J., Launonen, V., and Aaltonen, L.A. (2006). Analysis of fumarate hydratase mutations in a population-based series of early onset uterine leiomyosarcoma patients. Int J Cancer 119, 283-287.

Zaloudek, C., and Hendrickson, M.R. (2002) Mesenchymal tumors of the uterus, in Kurman RJ (ed): Blaustein`s Pathology of the Female Genital Tract (ed 5). New York, Springer-Verlag, pp561-578.

Zhai, Y.L., Kobayashi, Y., Mori, A., Orii, A., Nikaido, T., Konishi, I., and Fujii, S. (1999). Expression of steroid receptors, Ki-67, and p53 in uterine leiomyosarcomas. Int J Gynecol Pathol 18, 20-28. 